Revue d'histoire de l'Amérique française

Q REVUE D.HISTOIRE DE L'AMÉRIQUE FRANÇAISE

\title{
Chronique de la recherche
}

\section{Paul-André Linteau, Denis Bertrand, Pierre Savard, Fernand Harvey et Benoît Lévesque}

Volume 26, numéro 4, mars 1973

URI : https://id.erudit.org/iderudit/303224ar

DOI : https://doi.org/10.7202/303224ar

Aller au sommaire du numéro

Éditeur(s)

Institut d'histoire de l'Amérique française

ISSN

0035-2357 (imprimé)

1492-1383 (numérique)

Découvrir la revue

Citer ce document

Linteau, P.-A., Bertrand, D., Savard, P., Harvey, F. \& Lévesque, B. (1973).

Chronique de la recherche. Revue d'histoire de l'Amérique française, 26(4),

623-628. https://doi.org/10.7202/303224ar d'utilisation que vous pouvez consulter en ligne.

https://apropos.erudit.org/fr/usagers/politique-dutilisation/ 


\section{CHRONIQUE DE LA RECHERCHE}

Le Centre de recherche en civilisation canadienne-française de l'Université d'Ottawa - Fondé en 1958 dans le but d'étudier la littérature et transformé dix ans plus tard en centre interdisciplinaire (lettres, histoire et beaux arts), le CRCCF, dirigé par Paul Wyczynski, professeur titulaire à l'Université d'Ottawa, compte plusieurs réalisations à son actif. Sept collections regroupant des ouvrages de critique et de création littéraires et de bibliographie sont publiées sous son égide. Au nombre des réalisations intéressant les historiens, signalons le Projet Garneau qui prépare en 12 volumes une édition critique des Oeuvres complètes de l'historien national. Le Centre possède des Archives riches surtout en documents concernant l'histoire littéraire. Il a aussi recueilli une documentation considérable sur les sociétés patriotiques et culturelles franco-ontariennes.

Deux fois l'an depuis 1970 le CRCCF publie un Bulletin imprimé qui renferme des pages de création littéraire, de courtes études et des renseignements sur la recherche en cours; il est largement diffusé parmi les professeurs et les chercheurs. Ceux qui désirent le recevoir gratuitement n'ont qu'à envoyer leur nom et adresse à

Sœur Marguerite Charron,

Responsable du Bulletin,

Centre de Recherche en civilisation canadienne-française,

Université d'Ottawa,

Ottawa K1N 6N5.

Projet PRESARC - Les Archives Nationales du Québec à Montréal sont les dépositaires des greffes de 200 notaires pour 
la période $1647-1850$, ce qui représente environ 550,000 actes notariés. Plusieurs de ces greffes n'ont ni répertoire ni index, d'autres ont un répertoire mais il est totalement inutilisable. Cette situation fait qu'une bonne partie de cette riche documentation est difficile d'accès. Pour résoudre le problème un groupe a lancé le projet Préservation et aménagement des Archives Nationales du Québec à Montréal (PRESARC). Le coordonnateur en est Yves-Jean Tremblay qui a une vaste expérience en ce domaine. Le groupe a obtenu une subvention du gouvernement fédéral dans le cadre du programme d'initiatives locales ce qui a permis d'engager 34 personnes pour la plupart des étudiants et des jeunes diplômés. Le travail effectué est le suivant: dans une première étape, bâtir le répertoire de 87 greffes de notaires et le taper sur fiches; ensuite, améliorer les conditions de conservation des actes en les classant et en les protégeant individuellement. Ce travail considérable rendra de précieux services aux historiens qui sont de plus en plus conscients de la richesse documentaire des actes notariés. La subvention accordée se termine en mai 1973 et elle sera insuffisante. Les responsables estiment qu'elle devra être prolongée pendant une période de six mois pour que le projet soit réalisé entièrement.

PAUl-ANDré Linteau 
Congrès de l'ACFAS - Le 41e Congrès annuel de l'ACFAS se tiendra à l'Ecole Polytechnique de Montréal, les jeudi et vendredi, 24 et 25 mai 1973. Au programme de la section d'Histoire, on prévoit la présentation de communications sur des sujets aussi divers que "l'histoire de l'Hôtel-Dieu de Montréal" - "la condition ouvrière au Québec au début du 19e siècle" - "le parti ouvrier au Québec de 1899 à 1915" - "la consommation paysanne en France au 19e siècle" - "les structures familiales en Flandres vers 1800", etc. Il y aura également un "colloque", organisé par M. René Dal Magro avec la collaboration de M. Bruno Deshaies, et qui portera sur "le problème de l'enseignement de l'histoire à partir de l'étude de la "production historique" (épreuves, contrôles, travaux, examens) des étudiants du secondaire, du collégial et de l'universitaire". Une table ronde précédera des discussions en ateliers, à partir de "cahiers de travaux d'étudiants des 3 niveaux" que certaines équipes ont déjà commencé à colliger.

Pour recevoir le programme définitif du colloque, veuillez communiquer, par téléphone ou par lettre, avec: 
Monsieur Denis Bertrand

Université du Québec à Montréal

Département d'histoire

C.p. 8888

Montréal 101 tél.: 876-5525

Pour recevoir les cahiers des travaux d'étudiants, veuillez vous adresser à :

Monsieur René Dal Magro

Coordonnateur de l'enseignement de l'histoire au niveau collégial

Cegep Ahuntsic

9155, Saint-Hubert

Montréal 353 tél.: 389-5921

DENIS BERTRAND

secrétaire de la section

d'Histoire de l'ACF AS

Congrès 1973 
Trois instruments de recherche pour les chercheurs québécois en France -

ASSOCIATION' FRANÇAISE DE COOPÉRATION POUR L'HISTOIRE ET LA GÉOGRAPHIE, Recensement des unités de documentation d'histoire et de géographie. Edition préliminaire, Paris, Maison des Sciences de l'Homme, 1972. 2 volumes totalisant 500 pages. 451 unités décrites. Index des responsables. Index des matières. Index des sigles. Index des organismes. Préface de Jean Glénisson, directeur de l'Institut de recherche et d'histoire des textes.

Le répertoire décrit 451 organisations qui peuvent permettre la recherche documentaire (sources, études, répertoires) en histoire et en géographie. Il comporte deux parties: Paris et la région parisienne (231 notices) et la province française. Les notices comprennent les renseignements administratifs habituels (adresse, conditions d'accès...) et décrivent les domaines couverts. Bien utiles se révèlent les renseignements sur les collections particulières. Ce Recensement rendra les plus grands services aux chercheurs en leur évitant bien des pertes de temps et guidera sûrement leurs pas à travers la multiplicité des unités de documentation de l'hexagone.

L'Association française des Historiens et Géographes de langue française a subventionné cette publication réalisée par le Service d'Echange d'Informations scientifiques de la Maison des Sciences de l'Homme. 
Répertoire des bibliothèques et organismes de documentation. Direction des bibliothèques et de la lecture publique, Paris, Bibliothèque nationale, 1971. 733 p. Relié. Index. (Dépôt légal: 1972).

La Bibliothèque nationale de France vient de publier, sous la direction de Mlle Mireille Olivier, en collaboration avec Mlles Marie-Bernadette Julien et Jacqueline Mallet, un répertoire des bibliothèques et organismes de documentation. Il s'agit d'un travail considérable qui mérite d'être porté à l'attention de tous ceux qui poursuivent des recherches en France ou qui se proposent de le faire éventuellement. Ce répertoire vient compléter et mettre à jour le Répertoire des bibliothèques et organismes de documentation paru en 1963 sous l'égide de la Direction des bibliothèques de France. Cette nouvelle édition, publiée par la Bibliothèque nationale, présente 3,210 notices, dont 1,315 pour les organismes situés dans la région parisienne et 1,895 pour les organismes situés dans les autres départements.

Les divers dépôts d'archives, centres de documentation et bibliothèques recensés dans cet ouvrage sont susceptibles d'intéresser particulièrement les historiens, les sociologues et les divers spécialistes des sciences humaines et des lettres. Mais on y trouve aussi de nombreuses références aux sciences pures et aux techniques industrielles et commerciales.

Le répertoire comprend deux parties principales: le répertoire descriptif proprement dit et un index thématique. Le répertoire descriptif se subdivise en deux sections: région parisienne (classement. par ordre alphabétique de dénomination des unités repérées) et départements, y compris les départements et territoires d'outre-mer (classement par ordre alphabétique unique de communes). Les principales informations relatives à chaque unité de documentation concernent l'adresse complète, les heures d'ouverture, les conditions de prêt, les principales activités du centre et ses secteurs de spécialisation, de même que ses publications s'il y a lieu, un relevé statistique sommaire de ses collections, le type de classification et un bref historique depuis la fondation.

L'index thématique permet par ailleurs de repérer les diverses unités de documentation se rapportant à un même sujet dans le répertoire descriptif. Par exemple, au mot "Colbert", on trouve: - correspondance: Paris: Inspection du génie, 703. - fonds: mélanges: Paris: Bibliothèque nationale, Manuscrits, $237^{\mathrm{e}}$.

Cet instrument de travail, répétons-le, est indispensable à tous ceux qui se proposent d'entreprendre des recherches en France et qui veulent éviter de perdre un temps précieux. 
GADILLE, Jacques, Guide des Archives diocésaines françaises. Lyon, Centre d'histoire du catholicisme, 74, rue Pasteur, $1971,166 \mathrm{p}$.

Les archives diocésaines françaises sont souvent déconcertantes, au moins pour le chercheur étranger. En effet, si la plupart des fonds antérieurs à 1790 se retrouvent dans des dépôts départementaux, il existe cependant des exceptions. De même, si les fonds postrévolutionnaires considérés comme privés se retrouvent dans les archives diocésaines, il existe encore des exceptions. De plus, au feu et à la guerre, avatars communs à toutes les archives, il faut ajouter, pour les archives diocésaines, le "droit successoral qui livra aux héritiers, sans tri préalable, les papiers de tel évêque ou vicaire général". Dans ce contexte, on comprend l'intérêt du présent Guide des Archives diocésaines françaises.

La dédicace de ce recueil à la mémoire de Gabriel Le Bras constitue plus qu'un simple hommage à celui qui a relancé les études de sociologie et d'histoire religieuse en France puisque ce guide est le résultat d'une "enquête d'extension nationale décidée par la Société d'histoire ecclésiastique de la France en mai 1954". Le dépouillement définitif de cette enquête effectuée en 1964-65 ne fut réalisé qu'en 1968-69. Ce sont ces résultats que Jacques Gadille nous présente.

L'ouvrage est divisé en deux parties: l'une synthétique (pp. 7-40) et l'autre analytique (pp. 39-137). La première partie nous fournit ainsi le cadre général de cette recherche et les principales conclusions qui s'en dégagent. Ces conclusions sont regroupées autour de trois thèmes: sociologie du clergé, sociologie du peuple chrétien et histoire de l'institution ecclésiastique. La deuxième partie nous présente un inventaire analytique des fonds classés par diocèses. Parmi les noms des 83 diocèses qui se succèdent les uns aux autres selon l'ordre alphabétique, on remarque l'absence de Cambrai, Chambéry, Moutiers-en-Tarentaise et Saint-Dié. A l'intérieur de chaque diocèse, les divers fonds sont regroupés sous six titres: sociologie du clergé et du peuple chrétien, organisation diocésaine et liturgie, enseignement religieux, spiritualité, communautés religieuses et histoire générale.

Cinq annexes terminent l'ouvrage (pp. 139-164). Deux d'entre elles permettent une meilleure utilisation de l'inventaire analytique: liste alphabétique des saints ou bienheureux et des sanctuaires cités, index des papiers personnels conservés dans les archives diocésaines. Parmi les annexes relevons également le "cadre de classement proposé pour les archives diocésaines" qui nous rappelle que la décision de 1954 visait non seulement à recueillir des informations mais aussi à améliorer les conditions de ces archives. C'est pourquoi ce guide pourrait être utilisé avec avantage par les archivistes diocésains. Et cela d'autant plus que l'ouvrage de Jacques Gadille nous révèle qu'une telle entreprise ne saurait être réalisée sans la collaboration des archivistes eux-mêmes. 
Pour les chercheurs, retenons une double remarque de l'auteur sur les archives diocésaines, remarque qui en dernière analyse donne l'intérêt et la limite de son ouvrage: l'information contenue dans les archives diocésaines dépasse parfois le cadre de l'histoire religieuse pour atteindre celui de l'histoire générale mais ces archives ne sauraient suffire à l'histoire religieuse puisqu'elles n'en sont qu'un lieu parmi d'autres. Pour le Québec, nous pourrions ajouter qu'il semble de plus en plus difficile d'étudier, par exemple, le développement des communautés religieuses québécoises ou la formation des idéologies au XIX ${ }^{\mathrm{e}}$ siècle sans visiter les archives diocésaines françaises. Et pour cette visite nous ne pouvons que recommander le guide de Jacques Gadille. 